\title{
Compartir experiencias entre mujeres, artistas y docentes a través de herramientas virtuales.
}

\section{Share experiences among women, artist and teachers through virtual tools.}

\section{Resumen}

En este escrito cuento la experiencia que fue investigar desde un contexto online. Esta herramienta virtual me permitió compartir, dialogar y comprender las experiencias de un grupo de mujeres, artistas y docentes en Chile, transformándose en un lugar de encuentro y reflexión sobre el desarrollo profesional. Para explicar el proceso de investigación me centraré en dos aspectos importantes. Lo primero será contar cómo surgió y se realizó la plataforma. Por otro lado realizaré una breve revisión de la literatura sobre los métodos de investigación online para comprender, cómo se articularon, desde esta metodología virtual, las nociones de espacio, tiempo, y ética en este primer acercamiento a sus experiencias. Por último cuento mi rol y experiencia como investigadora insider-outiser y las relaciones que se construyeron en y a través de la plataforma.

Palabras clave: Escucha; experiencia compartida; investigación online; mujeres.

\begin{abstract}
In this paper I share the experience of investigating from an online context. This virtual tool allowed me to share, discuss and understand the experiences of a group of women, art teachers in Chile, becoming a place of encounter and reflection on their professional development. To explain the research process I will focus on two important aspects. First I will explain how this platform emerged and how was performed. On the other hand I will perform a brief review of the literature of online methods, to understand how it articulated, from this virtual methodology, the notions of space, time, and ethics in this first approach to their experiences. Finally I will tell my role and experience as a researcher insider-outsider and the relationships that were built in and through the platform.
\end{abstract}

Keywords: Listening; shared experience; online research; women

\section{Introducción}

Comparto la experiencia que viví durante la primera etapa del trabajo de campo de mi investigación doctoral, la cual fue desarrollada a través de un espacio virtual. Creo importante compartirla ya que muestra nuevas formas de experiencia de investigación y de acercamiento al campo, la cual escapa de las formas tradicionales para pensar en la noción de "etnografía multi-sitio" que nos propuso George Marcus (1995). Tras estas ideas, Marcus no nos propone estudiar espacios virtuales, sino que nos invitó a despojarnos de la idea del campo como espacio físico y singular, y a comprender que más que investigar una cultura localizada es seguir a la gente, generar conexiones, 
asociaciones, y dar cuenta de las relaciones que se generan en y a lo largo del espacio (Falzon, M.A., 2005).

En este sentido, esta herramienta virtual facilitó un primer acercamiento etnográfico, el cual me permitió compartir, dialogar y comprender las experiencias de un grupo de mujeres, artistas y docentes en Chile. Recalco que fue una primera aproximación ya que se desarrolló antes de comenzar el trabajo de entrevistas presenciales y de acompañamientos a sus prácticas docentes en Santiago de Chile. Este primer acercamiento, desde un contexto online, me permitió desde la distancia geográfica que nos separaba, comenzar a conocerlas y explorar las experiencias de las colaboradoras que conforman esta investigación.

Para acercarme a conocer las experiencias de las cuatro profesoras que colaboran en este estudio, construí una plataforma virtual llamada MujeresArtistasDocentes, en la cual generé un espacio personal para cada colaboradora (incluyéndome a mí como investigadora), que nos permitiera pensarnos y escribir nuestras experiencias como mujeres, artistas y docentes. A través de este espacio virtual se generaron instancias de diálogo, cruces y resonancias entre las colaboradoras, a partir de comentarnos las diversas experiencias. Es importante recalcar que mi rol como investigadora no fue de simplemente observar las experiencias que las colaboradoras compartían, sino que desde el inicio me configuré como parte del grupo, por lo que en la plataforma participé del mismo modo que ellas lo hicieron. Este posicionamiento me llevó a constantemente cuestionar qué significa e implica investigar como parte de un grupo online.

Durante tres meses, esta plataforma actuó como un espacio de reflexión asincrónico y como herramienta que permitió concretar los temas y preguntas que guían el trabajo que desarrollo actualmente con el grupo de profesoras. Todos los relatos de experiencias que se generaron en la plataforma, fueron la base de las entrevistas personales que se desarrollan de manera presencial en la segunda parte del trabajo de campo en Chile.

Para situar este primer acercamiento al trabajo de campo, en este escrito explicaré dos aspectos importantes sobre esta investigación online. La primera será contar cómo surgió y se realizó la plataforma. Por otro lado realizaré una breve revisión de la literatura sobre los métodos de investigación online y la Investigación Mediada por Internet (IMR) para analizar cómo se articularon las nociones de espacio, tiempo, y ética en este primer acercamiento a sus experiencias. Por último cuento $\mathrm{mi}$ rol y experiencia como investigadora insider-outiser y las relaciones que se construyeron en y a través de la investigación online con las profesoras. 


\section{Plataforma virtual “MujeresArtistasDocentes"}

Esta plataforma surgió desde la necesidad, como investigadora, de comenzar a acercarme a la vida y experiencia de las cuatro profesoras que conformarían mi investigación. Como investigadora interesada en comprender relatos de experiencia en la vida cotidiana, no podía permitirme tantos meses leyendo textos teóricos sobre estas nociones, y quizás simplemente porque las experiencias que quería escuchar, ver y comprender estaban a miles de kilómetros. En esos momentos, la distancia se me presentó como un problema porque yo estaba viviendo en Barcelona y ellas en Santiago de Chile. Sabiendo que el trabajo de entrevistas presenciales y acompañamiento a sus prácticas docentes comenzaría en unos nueves meses más, comencé a pensar estrategias que me permitieran acercarme a ellas, comenzar a conocerlas y escuchar sus experiencias. Así fue como llegué a la idea de crear un espacio virtual en internet. Soñé un tipo de espacio que estuviera al alcance de todas y que no fuera complicado de utilizar. Que nos permitiera reflexionar y compartir estas experiencias entre todas, sin importar dónde estuviésemos. A este espacio lo llamé MujeresArtistasDocentes ${ }^{1}$ y lo pensé como un sitio íntimo, que nos permitiera compartir experiencias, reflexiones y diálogos entorno a nuestros tránsitos entre la práctica artística y docente.

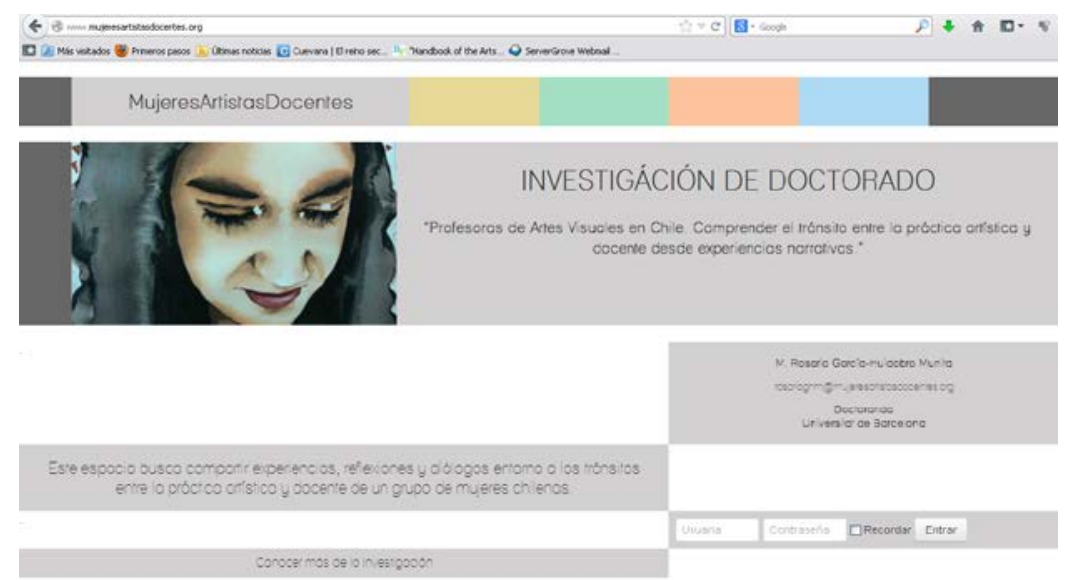

\section{Figura.01 Portada plataforma virtual MujeresArtistasDocentes}

Este espacio virtual se transformó en una herramienta que me permitió concretar los temas y preguntas que guiaron mi trabajo presencial con el grupo de profesoras en Chile. Por ello, ésta se sitúa como el primer acercamiento etnográfico de mi trabajo de campo.

\footnotetext{
${ }^{1}$ El espacio de investigación virtual fue creado en el sitio web www.mujeresartistasdocentes.org, como un espacio web privado, al cual sólo podíamos ingresar las cuatro colaboradoras y yo. Esta decisión surgió desde mi posicionamiento ético como investigadora. Si el objetivo de este espacio era compartir experiencias personales, entonces se volvía importante que el espacio fuera íntimo, seguro y confidencial. Sin embargo, es importante mencionar que al ser un sitio web, lógicamente existe una primera portada de acceso que es pública y a la cual todos tienen acceso.
} 
En esta primera etapa de investigación me centré en comprender sus experiencias y cómo se perciben a sí mismas como artistas y docentes, desde su sentido de ser mujer. A partir de las dimensiones: (1) ser mujer, (2) ser mujer artista, (3) ser mujer docente y (4) ser mujer artista docente, en la plataforma virtual compartimos diversas preguntas en relación a sus formaciones, prácticas y experiencias como artistas y docentes. Compartimos sus diversas creencias y concepciones sobre las artes y la enseñanza de las artes. También sobre el lugar que ocupan los otros en nuestra práctica artística y docente, etc. Todas estas preguntas han ayudado a comprender, desde sus voces y experiencias, cómo se perciben como mujeres en sus tránsitos entre las artes y la educación.

\subsection{Proceso de construcción del espacio virtual. Objetivos, descripción y funcionamiento de la plataforma.}

La planificación, diseño y construcción técnica de este sitio fueron construidos, en primera instancia, intuitivamente. Durante el proceso comencé a preguntarme qué significa, implica y cómo hacer una investigación virtual, por lo que acudí a revisar literatura sobre métodos de investigación online para comprender diversos aspectos metodológicos sobre este tipo de investigaciones y herramientas (en el siguiente apartado comento con mayor detalle esta literatura estudiada).

Para construir este sitio como un espacio virtual de investigación, primero concreté qué aspectos quería indagar y luego ver cómo y a través de qué herramientas y aplicaciones podría construirlo.

En este sentido, los objetivos que quería lograr a través de MujeresArtistasDocentes fueron principalmente dos: (1) Generar un espacio personal para cada colaboradora, que les permitiera pensarse a sí mismas y escribir sus experiencias en relación a cuatro temas específicos. (2) Generar instancias de diálogo, cruces y resonancias entre las colaboradoras, a partir de una opción de comentarnos las experiencias que fuésemos compartiendo.

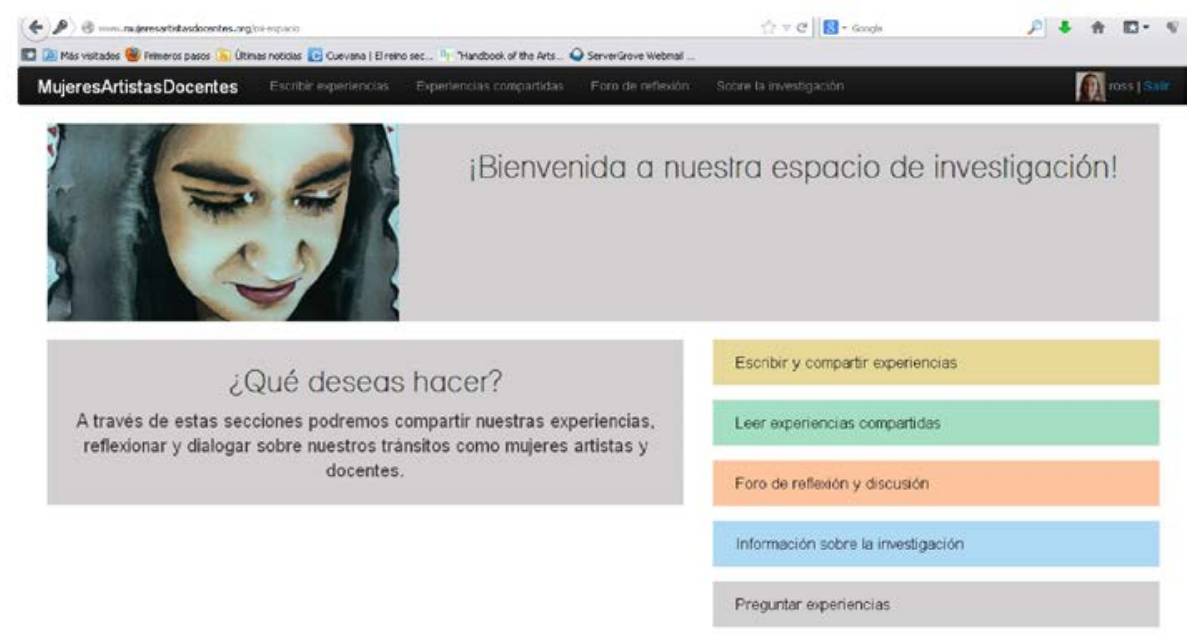

Figura.02 Home plataforma virtual MujeresArtistasDocentes 
De acuerdo a estos objetivos decidí enfocar este espacio a partir de cuatro temas o dimensiones individuales: (1) ser mujer, (2) ser mujer artista, (3) ser mujer docente y por último (4) ser mujer artista docente. Desarrollé cada tema de manera independiente y en orden cronológico, es decir cada mes avanzábamos con un tema y las diversas preguntas abiertas que yacían en cada tema. Esto me ayudó a ordenar el tipo de preguntas y profundizar en cada una de estas dimensiones, antes de comenzar a ver cómo dialogaban en el último tema. Es importante recalcar que, a pesar de que los temas se desarrollaron de manera separada, inevitablemente éstos se entrecruzaron.

Una vez concretados los objetivos y las preguntas que guiarían el trabajo pensé en cómo construir el sitio virtual. Tras explorar varias plataformas de investigación online ${ }^{2}$, no hallé ninguna que se ajustara a los requerimientos que tenía. Por ese motivo opté por desarrollar mi propio sistema adaptado y personalizado. Podría caracterizar a mi herramienta como una "opción híbrida" (Best, S.

y Krueger, B., 2008), en el sentido de que es una mezcla de las herramientas mencionadas por Claire Hewson (2007), sólo que adaptadas al funcionamiento y a los objetivos que tenía previsto.

A continuación explicaré qué son los métodos de investigación online y la importancia que han adquirido en los estudios cualitativos.

\section{Métodos de investigación online}

Para acercarme a los diversos métodos de investigación en red, fue importante comprender la importancia de internet como tecnología que nos ha permitido estas herramientas. Hoy en día podemos hablar de Internet como un espacio para la socialización, como "un cúmulo de conocimientos, un medio de comunicaciones, un foro comercial y un lugar para las experiencias" (Hine, C., 2001: 30). Éste se ha conformado como una cultura y también como un artefacto cultural que es representado en un lugar virtual que llamamos ciberespacio.

Hacia los años 90, Internet comenzaba a cobrar presencia pública como una herramienta que generaba redes sociales y por ende, nuevos conocimientos y formas de comprender la realidad. Por otro lado las tecnologías que ofrecía, atrajo a investigadores desde diversas disciplinas de las ciencias sociales (Fielding, N., Lee, R., Blank, G., 2008), quienes desafiados con la búsqueda de nuevas herramientas o métodos de estudio, comenzaron a explorar Internet para llevar a cabo investigaciones cualitativas (Meho, L., 2006). Frente a esto, Chris Mann y Fiona Stewart (2000) nos cuentan que las investigaciones de y a través de Internet no

\footnotetext{
${ }^{2}$ Los tipos de herramientas para investigaciones online y asincrónicas que ha señalado Hewson, C. (2007) son principalmente, e-mail, Listserv, Usenet, Bulletin Board System (BBS) y World Wide Web (WWW) forum.
} 
sólo tienen que ver con el estudio del comportamiento online, sino también con el uso de estas herramientas para estudiar el comportamiento humano.

Internet se ha convertido en un importante recurso de datos de estudio para las investigaciones en ciencias sociales. No sólo se trata de un lente para observar los sujetos que investigamos y el cómo construyen sus identidades y comunidades online, pero también puede ser una herramienta para la recopilación y el análisis de los datos en ciencias sociales (Eynon, R., Fry J., Schroeder, R., 2008: 26).

La utilización de Internet como herramienta de estudio no es una metodología que se ha comenzado a explorar recientemente (Mann \& Stewart, 2000), sino que también se ha configurado como un terreno variado, complejo y de rápidos cambios. Así mismo lo afirman Samuel Best y Brian Krueger (2008: 234), quienes también plantean que "Internet ofrece una gran variedad de oportunidades para recolectar datos de estudio". En este sentido, Claire Hewson y Dianna Laurent (2008) plantean que la Investigación Mediada por Internet (Investigation-Mediated Research) o IMR, refiere al tipo de investigación que utiliza a Internet para reunir datos y evidencias, las cuales 'al ser sometidas a un posterior análisis', develan nuevas evidencias en relación a la pregunta que investigamos.

Desde aquí, Hewson (2007) plantea una serie de herramientas para la IMR, dentro de las cuales encontramos la utilización del e-mail, Listserv, Usenet, Bulletin Board System (BBS), World Wide Web (WWW) forum, etc. Si bien la herramienta de investigación que yo utilizo no se basa en ninguna de las ya mencionadas, las nombro para mostrar la diversidad de medios que se utilizan para llevar a cabo investigaciones desde el ciberespacio.

Otra forma para conducir investigaciones a través de Internet, es la que Best y Krueger (2008) caracterizan como "opción híbrida" y frente a la cual me siento identificada. Éste es el caso de las investigaciones que se desarrollan a través de una página Web, y dónde además el contacto por email juega un papel importante de comunicación con los participantes. Encontrarme con que este "tipo de herramienta” ya fuese comprendida como válida dentro de la IMR, me ayudó a caer en la cuenta que había un lugar para aquello que yo estaba realizando.

Por otro lado, muchos investigadores señalan las ventajas que éstas herramientas nos entregan para investigar (Mann \& Stewart, 2000; Joinson, McKenna, Postmes y Reips, 2007; Fielding y Blank, 2008, entre otros). Como señala Hewson y Laurent (2008: 60), "la ventaja más obvia y que ha atraído a muchos investigadores, es la eficiencia del costo y el tiempo". Además, ha facilitado las 'investigaciones cross-cultural', es decir, nos ha permitido una mayor amplitud geográfica y traspasar límites culturales, aspecto que reafirma las ideas de George Marcus (1995) sobre etnografía multi-sited. Otra ventaja importante y destacada por muchos otros investigadores, es la que resumo en palabras de Henrietta $\mathrm{O}^{\prime}$ Connor, Clare Madge, Robert Shaw y Jane Wellens (2008: 273) 
Los entrevistados pueden responder las preguntas de la entrevista bajo su propia conveniencia. No hay restricciones de tiempo y esto puede ser particularmente valorable cuando los participantes están localizados en diferentes zonas horarias. La falta de restricciones temporales también permite al entrevistador y entrevistado, pasar tiempo considerando sus preguntas y respuestas, y quizás componiendo, recomponiendo y editando respuestas.

En este sentido, podemos comprender cómo este tipo de herramientas permite respuestas más profundas, reflexivas y detalladas.

También quisiera destacar que estas herramientas permiten que las relaciones de poder entre investigador y participantes sean más balanceadas (Hewson, 2007). Como se plantea en Steve Jones (1995), los ordenadores generan anonimato y este anonimato otorga el mismo estatus a todos los participantes, incluso los investigadores participantes. Sin embargo, a pesar de las diversas ventajas que entregan estas herramientas es importante reconocer y destacar que también existen aspectos que se diferencian de las investigaciones presenciales. Por ejemplo, la falta de visualidad entre investigador e investigado, mayor dificultad para generar relaciones, entre otras. Muchos de estos aspectos son tomados en cuenta en las IMR y han generado largos debates sobre las diferencias entre investigaciones online u offline (Mann \& Stewart, 2000; J oinson, McKenna, Postmes y Reips, 2007; Fielding y Blank, 2008, entre otros).

Debido a las preocupaciones y debates que estos aspectos han supuesto para las investigaciones mediadas por Internet, a continuación explicaré cómo desarrollé los componentes fundamentales de las IMR en mi investigación.

\section{Espacio, tiempo, ética, relaciones y rol del investigador en MujeresArtistasDocentes.}

Los componentes fundamentales de las Investigaciones mediadas por Internet son las nociones de espacio, tiempo, ética, relaciones y rol del investigador. Éstas, al igual que en las investigaciones presenciales contantemente se están cuestionando. En este sentido, el hecho que la IMR utilice un nuevo formato o bien el "campo" ya no sea un espacio físico sino virtual, me llevó a prestar mayor atención a cada uno de estos componentes, para comprender cómo se fueron desarrollando en mi investigación online.

\subsection{El espacio MujeresArtistasDocentes}

Quisiera iniciar esta reflexión contándoles que las preguntas, qué es MujeresArtistasDocentes y cómo se ha conformó este espacio en mi investigación, han sido cuestionamientos constantes. 
Desde las palabras de Hine (2001) y Jones (1995) comprendí que esta comunidad no poseía realmente un espacio físico de investigación, sino que un espacio virtual generado por redes y relaciones sociales que sólo y únicamente cobraba vida o dábamos sentido en los momentos que escribíamos, reflexionábamos y compartíamos. En este sentido, debido a las relaciones que se fueron generando en este sitio, más que simplemente ser una herramienta de investigación se transformó en nuestro lugar de encuentro. En un espacio virtual donde cada semana se iban generando y conectando nuevas experiencias, y por ende, donde semanalmente este espacio cobraba nuevos sentidos y significados.

Contarles cómo este espacio se articuló a partir de las interacciones, me traslada a las ideas de 'espacio relacional' y 'espacio-tiempo' de Doreen Massey (2005). Para esta autora el espacio está constantemente en proceso, porque siempre hay relaciones y conexiones que están por construirse. Sus palabras me llevaron a pensar esta plataforma de investigación como un espacio de trayectorias simultáneas, cargada de historias y experiencias vividas, de conexiones, sentidos y sin sentidos. Este espacio como un lugar incompleto, inacabado, que cada día estaba a la espera de nuevas relaciones que lo fueran cargando de sentidos y recorridos.

En este sentido, aparte de que este espacio estaba en constante conformación, también estaba mediado por múltiples conexiones. Pensemos que cada una de nosotras entraba al sitio de manera separada y luego escribía individualmente su propia experiencia en su sitio personal. Pero, a pesar de que cada una tuviera su propio espacio, existía un espacio común donde se entrecruzaban todas las experiencias y sentidos. Nuestras relaciones se conectaban y a su vez, nosotras mismas las íbamos re-conectando y cargando de nuevos sentidos cuando leíamos las experiencias de las otras y compartíamos nuevas experiencias entre todas. Se generaba un diálogo múltiple que, si bien se originaba de manera individual, poco a poco se iban sumando voces que lo transformaban en un diálogo entrecruzado de diversas experiencias femeninas sobre las artes y la educación.

\subsection{El factor tiempo en MujeresArtistasDocentes}

Doreen Massey (2012) nos señala que todo espacio contiene tiempo en su interior. Frente a esta afirmación y mirando cómo éste se fue articulado en mi espacio de investigación, puedo decir que el tiempo fue un factor fundamental, ya que permitió generar las interacciones, conformando a nuestro espacio como un lugar abierto a los contantes cambios y transformaciones.

En este sentido, en mi investigación busqué generar interacciones sociales que no fueran acordes al tiempo real, es decir, asincrónicas. Escogí este tipo de relación pensando en diferentes aspectos. Lo primero fue ligado a nociones prácticas como la diferencia horaria entre Santiago y Barcelona (entre 4 y seis horas dependiendo de la época) y a sus propias cargas horarias (colegio, vida personal, otras actividades, etc.). Frente a estos aspectos pensé, ¿por qué exigirles o imponerles 
un horario para estar en el ordenador? Lo segundo y ciertamente muy importante, fue que prioricé que pudieran entrar a escribir y compartir al sitio cuando quisieran. Es decir, que se sintieran libres de acceder y escribir cuando estimaran conveniente, sin "presiones de tiempo". Pensemos que cada domingo yo colgaba una pregunta y cada una de nosotras tenía toda una semana para pensar, escribir y visitar las narraciones de las otras. Frente a esto, me sumo a las experiencias de otros académicos que investigando con herramientas asincrónicas dicen haber generado datos más ricos, detallados, elaborados y reflexivos (Murray y Sixsmith, 1998; Bowker y Tuffin, 2004; Hewson y Laurent, 2008; Hewson, 2007). En mi caso, el hecho que cada una pudiera escribir con tranquilidad provocó que las experiencias compartidas tuvieran una gran carga reflexiva. Sus respuestas eran dedicadas y detallas, lo que demostraba no sólo un compromiso con la investigación, sino también por desear reflexionar sobre sus propios recorridos como mujeres, artistas y docentes.

Finalmente, quisiera compartir las palabras que una de las participantes me señaló en dos instancias diferentes:

“Me parecen muy acertadas tus preguntas, una buena instancia para detenerse a reflexionar. Sólo quisiera tener más tiempo...." (Respuesta de correo el 28/10/2013)

“Ross, (...), en cuanto tenga un tiempo, contestaré tus preguntas, que están muy interesantes, pero necesitan un tiempo de reflexión. (Respuesta de correo el 15/10/2013)

Lo que quiero señalar a través de sus palabras, es cómo me daban a entender que las preguntas y temas que desarrollábamos les y nos exigía tiempo para pensar, decantar, mirar nuestra historia y la de otros. En este sentido, el tiempo asincrónico fue una herramienta que potenció a que cada una pudiera cuestionarse desde el lugar, contexto o situación desde dónde estuviésemos. Nos permitió pensarnos para generar nuestros propios sentidos y conexiones, y a su vez, abrirnos a las experiencias de las otras para generar nuevo sentidos y conexiones.

\subsection{La ética durante el proceso de investigación}

El factor ético ha estado presente desde los inicios en esta investigación. Situar mi estudio desde un enfoque feminista, me ha llevado a reflexionar cada paso que he ido dando. Cuestionar continuamente mi rol como investigadora ha transformado mi dinámica o proceso de investigación, en un juego constante, de avanzar y retroceder. La ética de las investigadoras feministas me ha señalado dos aspectos fundamentales a tener en cuenta, los cuales yo me he apropiado para conformar mi propio sentido de investigación. La primera es considerar la importancia del "otro" en mi investigación (The personal Narrative Group, 1988). En mi caso, hablamos que ese "otro" son mujeres, artistas y docentes como yo, y por ende, a pesar de las diferencias que podamos sostener, existe algo que nos compromete y compenetra como grupo. Otro aspecto importante ha 
sido la invitación a comprender que aquello que me sucede como mujer investigadora es parte de la investigación, y por ende, he aprendido a compartir y develar mi propio proceso de investigación. Estos dos aspectos han sido fundamentales no sólo para comprender desde dónde me sitúo para investigar, más bien para configurar cuáles son esos valores que orientan mi sentido de investigación.

Por otro lado, quisiera recalcar tres aspectos éticos importantes, los cuales fueron importantes durante el proceso de trabajo en la plataforma. En primer lugar y paralelo a la plataforma, el correo electrónico fue una herramienta que nos mantuvo en constante comunicación y relación. A través de éste, siempre mantuve un vínculo más personal con cada una, es decir, nunca envié correos grupales. Opté por escribirlos de manera personalizada para intentar ser más cercana y afectiva con cada una. Mediante esta vía no sólo les envié los informes de confidencialidad o las cartas para acceder a sus instituciones educativas, sino que además todo ello fue un proceso de negociación que implicó un período de conversaciones por e-mail. Por ejemplo, negociar aspectos de identidad y anonimato o hasta qué punto estaban dispuestas a colaborar, fueron temas importantes y de constante negociación antes de comenzar con la plataforma.

En segundo lugar, preguntarme por el factor ético de la plataforma virtual fue fundamental e implicó un proceso de creación especializado. Si deseaba que éste fuera un espacio íntimo, donde compartiéramos experiencias personales, entonces técnicamente debía ser seguro y confidencial, lo que implicó diseñar herramientas de login y clave personal. Por otro lado, deseaba que el sitio visualmente les pareciera acogedor y de fácil uso, para que se sintieran cómodas e inspiradas al escribir. Por ello, el diseño de las imágenes, tipografías y estructura fueron aspectos que desarrollé con precaución. Otro aspecto ético importante de la plataforma fue la decisión de que cada una ingresara a su sitio personal. Es decir, si bien existía una sección común para leer las experiencias compartidas por todas, la sección para escribir era íntima y además, cada una decidía si compartía su respuesta sólo conmigo o bien con todas. Esto lo pensé porque deseaba que cada una pudiera narrar tranquila y en su propio espacio. Además, que dependiendo del nivel de apertura de las respuestas, ellas pudieran escoger si lo compartían o no. Frente a esto debo señalar que la mayoría de respuestas fueron compartidas entre todas, sólo unas pocas fueron enviadas únicamente a mí ya que como ellas mencionaban 'contenían un alto grado de intimidad'.

\subsection{Mi rol como investigadora 'in bettween'}

A partir del Interpretativismo, "la participación es el ingrediente característico del trabajo de campo antropológico" (Guber, R., 2004: 119). En este sentido, como nos plantea Rosana Guber (2004), la observación participante consistía en dos actividades. La primera era observar 'sistemática y 
controladamente' todo aquello que sucedía alrededor del investigador y por otro lado la actividad de participar, que consistía en vivir y comportarse como uno más de la comunidad.

Esta descripción sobre la 'participación con observación' también ha sido tema de interés en las investigaciones mediadas por Internet (Taylor, J. 2011). Muchos investigadores online se han preguntado qué significa y cómo ser un observador participante en una comunidad virtual (Paechter, C., 2012). En este sentido, en las IMR podemos encontrar que el investigador puede posicionarse de dos formas. La primera es llamada "Lurker" y describe al investigador que simplemente observa la comunidad. Puede acceder a todo el contenido de la comunidad virtual, pero no participa. Algunos investigadores argumentan que este tipo de posicionamiento no es suficiente, ya que al no existir una interacción con los participantes, el investigador se pierde cosas, aspectos o situaciones. Por ello, otros investigadores argumentan que "la participación activa es esencial para acceder a las experiencias vividas de los miembros de la comunidad" (Paechter, 2012: 74). Cuando un investigador participa en una investigación online, el investigador interactúa como miembro de la comunidad. Esto puede suceder a través de su participación en foros, chats, entrevistas, etc. Como comparte Tom Boellstorff (2012) a través de su experiencia como etnógrafo de mundos virtuales,

La observación participante se basa en las alineaciones entre, por un lado la participación en las actividades cotidianas, y por otro grabando y analizando esas actividades. El truco de esta alineación a veces nos lleva a preguntar, “¿Estás jugando o investigando?”. El bien o naturaleza de esta cuestión olvida que la observación participante significa que participarincluyendo el juego- es absolutamente esencial. No podemos escoger una o la otra. Una buena observación participante significa jugar e investigar en paralelo, como la misma actividad. (Boellstorff, 2012: 69)

A raíz de las palabras de Boellstorff, pienso en la investigación etnográfica que publicó el 2008. Tom llevó a cabo su investigación como un avatar y desde su participación activa en el mundo virtual Second Life, intentó comprender cómo otros viven la cotidianidad virtualmente. Lo que me sorprendió de su trabajo no fue la metodología, ya que como antropólogo aplicó rigurosamente los métodos tradicionales de la etnografía. Más bien fue preguntarme por su rol. Si para entrar a investigar en el juego, Tom Boellstorff se creó un avatar llamado "Tom Bukowski", me pregunto ¿cómo negoció o lidió entre este juego de identidades? En base a su experiencia, no me surgen preguntas vinculadas a su rol como observador participante, sino más bien con el significado de investigar una comunidad de la cual formamos parte y de negociar nuestros roles dentro y fuera de la investigación. Estas preguntas cobran sentido con mi propio rol como investigadora y me llevan a pensar en el posicionamiento inside-outside, como una forma de cuestionar y reflexionar nuestro propio posicionamiento como observadores participantes. 
Acercarme a comprender las formas en que se han cuestionado los posicionamientos del investigador en ciencias sociales y el posicionamiento insider-outsider, me ha permitido repensar y comprender qué significa ser una investigadora que transita entre múltiples posicionamientos. Cuando refiero a múltiples posicionamientos, primero hago alusión a cómo he ido dialogando con mis roles como mujer artista, docente e investigadora, y luego cómo estos roles interactúan y se relacionan con las otras mujeres participantes, desde un medio de investigación online.

En este sentido, la decisión de no ser una "analista invisible" o una simple "lurker" (Hine, 2001) de la plataforma virtual se debe a diversas razones. La primera y más importante toca mi propio posicionamiento como investigadora feminista. Porque al mismo tiempo que soy investigadora soy mujer, soy artista y docente, y desde este punto, comprender las experiencias de otras mujeres artistas ha implicado involucrarme y no eludir mis propias circunstancias. Ahora bien, me detengo en este punto y remarco una diferencia. Kusow (2003) me enseñó que un investigador puede compartir muchas características con sus participantes, pero eso no determinará su posición insider, ya que ello dependerá de las relaciones que se formen y cómo se van negociando. En este sentido, no sólo compartir con ellas el ser mujer, artista y docente (a pesar de que no estar ejerciendo docencia como ellas en estos momentos), me ha llevado a sentirme y ser una más del grupo. Sino más bien la relación que durante el proceso fuimos generando. Ser observadora participante o bien participante activa, y reflexionar, escribir y compartir al igual que las otras profesoras, ha sido fundamental para la relación que fuimos formando, y por ende, el posicionamiento que fui tomando.

Por otro lado, si bien participar activamente de la plataforma significó, al igual que ellas, reflexionar y narrar semanalmente mis propias experiencias, también me percaté que con algunas preguntas mi posicionamiento insider era re-cuestionado. En este proceso, caía en la cuenta que, al igual como me señalaban algunas investigadoras feministas (Dwyer and Buckle, 2009; Taylor, 2011), nunca era completamente una insider, ya que habían ciertos aspectos que como investigadora me condicionaban o limitaban. Por ejemplo, el hecho de no estar ejerciendo docencia en esos momentos, me impedía contar aspectos sobre mi práctica docente actual, por lo que en esos casos recurría a compartirlas más desde mi experiencia pasada. Frente a este tipo de situaciones, si bien ellas no me hacían sentir menos parte del grupo yo era quien me apartaba y notaba que mi posicionamiento estaba, al igual como lo experimentan Dwyer and Buckle (2009) en un espacio entre, es decir, 'in bettween'.

A partir de la experiencia de investigar online y ser parte del grupo que investigaba, comprendí que mi rol como investigadora en la plataforma no estaba en una localización fija, sino que iba fluyendo. En este tránsito me iba desplazando dentro y fuera. Estaba fuera cuando semanalmente colgaba las preguntas en la plataforma, pero a su vez estaba dentro cuando esas mismas 
preguntas que enviaba, las reflexionaba y compartía. Estaba fuera cuando en muchas preguntas no sabía desde qué lugar escribir, pero estaba dentro cuando veía respondían mis comentarios y dialogábamos sobre sus y mis experiencias. Durante este proceso fui lidiando y negociando con mis múltiples roles y posicionamientos dentro de la investigación.

\section{Conclusión}

En este escrito he contado la experiencia que fue investigar, durante tres meses, desde un contexto online. Esta herramienta virtual se transformó en un lugar de encuentro y reflexión sobre sus y mis diversas experiencias. La plataforma permitió abrir un espacio de re-interpretación, desde el acto de relatarnos, como estrategia que permitió dar nuevos significados a nuestras experiencias como mujeres, artistas-docentes. Así también recuperar sus experiencias de formación artística y docente y ponerlas en diálogo con las experiencias de las otras participantes, permitió repensar y cuestionar en conjunto, los diversos discursos y convenciones sociales sobre "mujer artista" y "mujer docente".

Por otro lado, compartir estas experiencias a través de la plataforma permitió visibilizar los diversos modos en cómo cada una significa personal y socialmente sus propios recorridos como artistas y docentes y, por ende, desvelar que el tránsito entre la práctica artística y docente son recorridos de experiencia y conocimiento que se articulan y construyen mutuamente.

\section{Referencias bibliográficas}

Best, S., Krueger, B. (2008) Internet Survey Design. En Fielding, N., Lee, R., Blank G. The SAGE Handbook of Online Research Method. (pp. 217-236) London: SAGE.

Boellstorff, T., Nardi,B., Pearce, C., Taylor, T. L. (2012) Ethnography and Virtual Worlds: A Handbook of Method. New Jersey: Princeton University Press

BOWKeR, N., TUFFIN, K. (2004) Using the Online Medium for Discursive Research About People With Disabilities. Social Science Computer Review, 22(2), 228-241. SP

DWYER, S., BUCKLE, J. (2009) The Space Between: On Being an Insider-Outsider in Qualitative Research. International J ournal of Qualitative Methods, 8(1), 54-63.

EYNON, R., FRY J., SChroeder, R. (2008) The Ethics of Internet Research. En Fielding, N., Lee, R., Blank G. The SAGE Handbook of Online Research Method (pp. 23-41) London: SAGE.

Falzon, M.A. (2009). Introduction. In M. Faixon (Ed.) Multi-sited Ethnography: Theory, Praxis and Locality in Contemporary Research (pp. 1-24). Cornwall: Ashgate

Fielding, N., LeE, R., BLANK, G. (2008) The Internet as a Research Medium: An Editorial Introduction to The Sage Handbook of Online Methdos. The SAGE Handbook of Online Research Method (pp. 3- 20) London: SAGE.

GubBeR, R. (2004) El salvaje metropolitan. Reconstrucción del conocimiento social en el trabajo de campo. Buenos Aires: Paidós 
HEWSON, C., LAURENT, D. (2008) Research Design and Tools for Internet Research. En Fielding, N., Lee, R., Blank G. The SAGE Handbook of Online Research Method ( pp. 58-78) London: SAGE.

HINE, C. (2001) Virtual Etnography. London: SAGE

JoInson, A., McKenna, K., Postmes, T., Reips, U.D. (2007) The Oxford Handbook of I nternet Psychology. Oxford: University Press.

J OINSON A., PAINE, C. (2007) Self-disclosure, privacy and the Internet. En Joinson, A., McKenna, K., Postmes, T., Reips, U.D. The Oxford Handbook of Internet Psychology (pp. 237-252) Oxford: University Press.

J ONES, S. (1995) Cybersociety. Computer-Mediated Communication and Comunity. London: SAGE.

Kusow, A. (2003) Beyond Indigenous Authenticity: Reflections on the Insider/Outsider Debate in Immigration Research. Symbolic Interaction, 26(4), 591-599.

MADGE C., O'CONNOR, H. (2002) On-line with e-mums: exploring the Internet as a medium for research. Royal Geographical Society, 34(1), 92-102

MAnN C., Stewart, F. (2000) Internet Communication and Qualitative Research: A Handbook for Researching Online. SAGE: London.

MARCUS G. (1995) Ethnography in/of the World System: The Emergence of Multi-Sited Ethnography. Annual Review ofAnthropology, 24, 95-117.

MASSEY, D. (2005) For Space. SAGE: California

MEHO, L. (2006) E-Mail Interviewing in Qualitative Research: A Methodological Discussion. J ournal of the American Society for I nformation Science and Technology, 57(10), 1284-1295.

MURRAY C D, SIXSMITH J. (2008) Email: a qualitative research medium for interviewing? International J ournal of Social Research Methodology: Theory and Practice, 1(2), 103-121.

PAECHTER, C. (2012) insider/outsider position in a retrospective ethnographic study Researching sensitive issues online: implications of a hybrid. Qualitative Research, 13(1), 71-86.

TAYLOR, J. (2011) The intimate insider: negotiating the ethics of friendship when doing insider research. Qualitative Research, 11(1), 3-22.

The Personal Narrative Group (1989) Interpreting Women's Lives. Feminist Theory and Personal Narratives. Indiana: Indiana University Press.

Rosario García

Huidobro Munita

Recebido em: 25/07/2014

Aprovado em: 08/08/2014 